\title{
MAXIMAL CONNECTED EXPANSIONS OF THE REALS
}

\author{
J. A. GUTHRIE ${ }^{1}$, H. E. STONE AND M. L. WAGE
}

\begin{abstract}
The question of whether there exist nontrivial maximal connected Hausdorff spaces is settled in the affirmative by showing that there is a maximal connected topology for the reals which is finer than the Euclidean topology.
\end{abstract}

1. Introduction. Much of the interest in the lattice of topologies on a set has centered on those topologies which are maximal or minimal with respect to some topological property. In this paper we show that there exist nontrivial Hausdorff topologies maximal with respect to connectedness. This answers a question first raised by Thomas in 1968 [15]. The existence of the question has been noted by other authors [11], [14].

Progress on the problem has taken two directions. One approach has been to derive the properties that a maximal connected Hausdorff space must have [15], [16], [6], [7], [5] and to study those properties and examples of such spaces [10], [7].

The other approach has been to try to answer certain related questions. One such question is: Given a connected topology, how may a finer connected topology be constructed? We discuss this question in the next section. Another related question is: Does every connected Hausdorff space admit a finer connected topology which is maximal connected? This question was answered in the negative by Guthrie and Stone [7], who showed that no connected Hausdorff space with a dispersion point has a finer maximal connected topology; and by Baggs [2], who studied an example of such a space. A third related problem was raised in 1968 by Hammer and Singletary [8] independently of Thomas: Does there exist a maximal connected topology finer than the usual topology on the reals? In this paper we answer this question, and hence Thomas' more general question, in the affirmative. Solutions were obtained independently by Wage and by Guthrie and Stone. The constructions are different, and both will be described.

Let us fix some terminology and notation. We say that a space $(X, \sigma)$ is an expansion of $(X, \tau)$ if $\sigma \supseteq \tau$. In this case we also call $\sigma$ an expansion of $\tau$. The expansion of the topology $\tau$ on the set $X$ by a collection $\mathscr{Q}$ of subsets of $X$ is

Received by the editors May 4, 1977 and, in revised form, September 23, 1977.

AMS (MOS) subject classifications (1970). Primary 54A10, 54D05, 54G15.

Key words and phrases. Maximal connected, expansion of a topology, lattice of topologies.

${ }^{1}$ Partially supported by a URI grant from the University of Texas at El Paso. 
the topology with subbase $\tau \cup \mathbb{Q}$, and it is denoted by $\tau(\mathbb{Q})$. In case $\mathbb{Q}=\{A\}$ we write $\tau(A)$ in place of $\tau(\{A\})$. Throughout $R$ will denote the set of real numbers, $I$ the set of points in the closed unit interval, $Q$ the rationals, and $\varepsilon$ the usual Euclidean topology for $R$ or $I$.

We denote the boundary and interior of a set $A$ with respect to $\tau$ by $\partial_{\tau} A$ and $\operatorname{Int}_{\tau} A$, respectively. The cardinality of the set $A$ is represented by $|A|$, and $c$ is $|R|$.

2. Expansion of topologies. The major class of expansions which have been known to preserve connectedness consists of those obtained by adjoining a filter of dense sets. Results of this type have been obtained by several authors [1], [3], [4], [6], [10], [12], [13]. Bourbaki [4] points out that these are exactly the expansions which leave fixed the semiregular compression $\tau_{s}$ of $\tau$, the topology generated by the regular open sets of $\tau$. The semiregular compression was introduced by Katetov [9], who showed that it leaves invariant all continuous functions on the space which take values in a regular space; from this the connectedness preserving property of dense-filter expansions is immediate.

If $\tau$ is expanded by an ultrafilter of $\tau$-dense sets, the resulting topology is called submaximal by Bourbaki [4]. Note that if $\sigma$ is a submaximal topology and $A$ is any subset of the space, then $\partial_{\tau} A$ is a discrete set.

Let $X \subseteq R$ and let $\tau \supseteq \varepsilon$. Then a subset $A$ of $(X, \tau)$ is biperfect if for each $a \in A, a \in \mathrm{Cl}_{\tau}(A \cap(-\infty, a)) \cap \mathrm{Cl}_{\tau}(A \cap(a, \infty))$. If $A \subseteq X$ is biperfect, then $A$ is singular at $p \in A$ if $A-\{p\}$ is $\tau$-open. An expansion $\sigma$ of $\tau$ is a singular expansion if every point $x \in X$ has a $\sigma$-nbd base which is a filterbase of sets $\tau$-singular at $x$. The expansion $\sigma$ of $\tau$ is maximal singular (over $\tau$ ) if for each $x \in X$ there is a $\sigma$-nbd base at $x$ which is an ultrafilter base of sets $\tau$-singular at $x$.

3. The construction of Guthrie and Stone. The first theorem establishes a certain compatibility of maximal singular expansions and expansions by filters of dense sets.

THEOREM 1. Let $\mathscr{D}$ be an ultrafilter of $\varepsilon$-dense sets and for each $x \in R$, let $\mathcal{G}_{x}$ be an ultrafilter of sets $\varepsilon$-singular at $x$. Let $\mathcal{G}=\cup_{x} \mathcal{G}_{x}$. Then there exists for each $x \in R$ an ultrafilter $\mathscr{F}_{x}$ of sets $\varepsilon(\mathscr{D})$-singular at $x$ such that $\varepsilon(\mathcal{G})(\mathscr{D})$ $=\varepsilon(\mathscr{D})(\mathscr{F})$, where $\mathscr{F}=\cup_{x} \mathscr{F}_{x}$.

Proof. For each $x \in R$, set $\mathscr{F}_{x}=\left\{(G \cap D) \cup\{x\} \mid G \in \mathcal{G}_{x}, D \in \mathscr{D}\right\}$. Clearly, $\mathscr{F}_{x}$ is a filterbase of sets $\varepsilon(\mathscr{D})$-singular at $x$. Suppose $V$ is a set such that $\mathscr{F}_{x} \cup\{V\}$ is a filterbase of sets $\varepsilon(\mathscr{D})$-singular at $x$. Then $V-\{x\} \in$ $\varepsilon(\mathscr{D})$, so there exists $W \in \varepsilon$ and $E \in \mathscr{D}$ such that $V-\{x\}=W \cap E$. Thus, for $b>x$,

$$
\varnothing \neq[(W \cap E) \cap(G \cap D)] \cap(x, b)=(W \cap G) \cap(E \cap D) \cap(x, b)
$$

for each $(G \cap D) \cup\{x\} \in \mathscr{F}_{x}$. Thus, $(W \cap G) \cap(x, b) \neq \varnothing$ for each $G \in$ $\mathcal{G}_{x}$. Similarly, $(W \cap G) \cap(a, x) \neq \varnothing$ for each $a<x$. Thus, $W \cup\{x\}$ is $\varepsilon$-singular at $x$ and has nonempty $\varepsilon$-singular intersection with each $G \in \mathcal{G}_{x}$. 
Thus, $W \cup\{x\} \in G_{x}$ and $V \in \mathscr{F}_{x}$. Hence, $\mathscr{F}_{x}$ is an ultrafilter of sets $\varepsilon(\mathcal{D})$-singular at $x$.

Now $\mathcal{G} \subseteq \mathscr{F}$, so $\varepsilon(\mathcal{G})(\mathscr{D}) \subseteq \varepsilon(\mathscr{F})(\mathscr{D})=\varepsilon(\mathcal{D})(\mathscr{F})$. Conversely, in $\varepsilon(\mathscr{D})(\mathscr{F})$ each point $x$ has a basis of the form $V \cap D \cap F$ for $V \in \varepsilon, D \in \mathscr{D}$, $F \in \mathscr{F}_{x}$, and $x \in V \cap D \cap F$. But $F=(G \cap E)$ for some $G \in \mathcal{G}_{x}, E \in \mathscr{D}$, so

$$
V \cap D \cap F=V \cap D \cap(G \cap E)=V \cap G \cap(D \cap E) \in \varepsilon(\mathcal{G})(\mathcal{D}) .
$$

Thus, $\varepsilon(\mathscr{D})(\mathscr{F}) \subseteq \varepsilon(\mathcal{G})(\mathscr{D})$ and the proof is complete.

Thus, an expansion of $(R, \varepsilon)$ which is obtained by a maximal singular expansion followed by a submaximal expansion may be obtained as a submaximal expansion followed by a maximal singular one.

Every topology admits maximal singular expansions, but in Example A below we show that singular expansions of $(I, \varepsilon)$ may be disconnected. We want to be able to construct connected ones.

THEOREM 2. Suppose $(I, \sigma)$ is a singular expansion of $(I, \varepsilon)$ and suppose $\{A, B\}$ is a partition of $(I, \sigma)$, i.e., illustrates that $(I, \sigma)$ is disconnected. Set $C=I-\left(\operatorname{Int}_{\varepsilon} A \cup \operatorname{Int}_{\varepsilon} B\right)$. Then $(C, \varepsilon)$ is a homeomorphic image of the Cantor set.

Proof. Clearly, $\operatorname{Int}_{\varepsilon} A$ and $\operatorname{Int}_{\varepsilon} B$ are nonempty and $C$ is nonempty, $\varepsilon$-closed, and totally disconnected. Let $x \in C \cap A$. Every interval $J$ about $x$ must contain points of $B$, but $\operatorname{Int}_{\varepsilon} B$ can be expressed as a disjoint union of at most countably many open intervals $B_{n}$. Thus, since $J \notin \operatorname{Int}_{e} B, J$ contains an endpoint of some $B_{n}$. But an endpoint of $B_{n}$ cannot be in $A$. Thus, each interval about $x$ contains a point of $B \cap C$. Similarly, each point in $B \cap C$ is an $\varepsilon$-cluster point of $A \cap C$. Hence $(C, \varepsilon)$ is perfect, and we are done.

This information about disconnected singular expansions allows us to exhibit one.

EXAMPLE A. Let $C$ be the usual Cantor set constructed by removing middle thirds from $I$. Let $A$ be the union of the closures of the intervals removed at odd numbered steps, and let $B=I-A$. For every $x \in C \cap A$ let $U_{x}=\{x\}$ $\cup \operatorname{Int}_{\varepsilon} A$. For $x \in C \cap B$, let $U_{x}=\{x\} \cup \operatorname{Int}_{\varepsilon} B$. Let $\mathcal{Q}=\left\{U_{x} \mid x \in C\right\}$. Then $\varepsilon(\mathcal{U})$ is a singular expansion of $I$ which is disconnected by $A$ and $B$.

THEOREM 3. There exists a connected maximal singular expansion of $(I, \varepsilon)$.

Proof. By a Cantor set in $I$, we mean a subset of $I$ homeomorphic to the usual Cantor set; i.e., a closed, perfect, totally disconnected subset. Let $g$ be the set of all complements of Cantor sets in $I$. Then each $J \in g$ is a union of countably many disjoint open intervals. Now $J$ is determined by the countably many endpoints of their intervals, so $|\mathscr{f}| \leqslant c^{\omega}=c$.

Now for each $J \in \mathcal{g}$ consider $\mathcal{C}(J,\{0,1\})$, the set of all continuous functions from $J$ to $\{0,1\}$. Each such function must be constant on each of the countably many disjoint open intervals into which $J$ can be decomposed. For each $J$ there are $2^{\omega}=c$ such functions. Let $\mathscr{B}=\{(J, f) \mid J \in \mathcal{g}, f \in$ 
$\mathcal{C}(J,\{0,1\})$ and $\left.I-J \subseteq \mathrm{Cl}^{-1}(0) \cap \mathrm{Cl} f^{-1}(1)\right\}$. Now $|\mathscr{B}| \leqslant c$, and for every $J \in \mathcal{G}, I-J$ is a homeomorph of the Cantor set and, hence, $|I-J|=$ c.

We now well-order $\mathscr{B}$ and index it by the least ordinal $\lambda$ whose cardinal is $|\mathscr{B}|$. We write $B_{\alpha}$ for the $\alpha$ th pair $(J, f)$. Thus, $\mathscr{B}=\left\{B_{\alpha} \mid \alpha<\lambda\right\}$. Similarly, we may well-order each $C=I-J$ and index it by the least ordinal whose cardinal is $c$, also denoted by $c$. Then $C=\left\{x_{\alpha} \mid x_{\alpha} \in I-J, \alpha<c\right\}$.

Next we construct a mapping $g: \mathscr{B} \rightarrow I$ by transfinite induction. For $B_{0}=(J, f)$, let $g\left(B_{0}\right)=x_{0}$, the first element of $I-J$. Assume that $g\left(B_{\alpha}\right)$ has been defined for each $\alpha<\beta$ such that $g\left(B_{\alpha}\right)$ is the first element of $I-J^{\prime}$ not previously chosen, where $B_{\alpha}=\left(J^{\prime}, f^{\prime}\right)$.

Now define $g\left(B_{\beta}\right)$ to be the first element of $I-J^{\prime \prime}$ not previously chosen, where $B_{\beta}=\left(J^{\prime \prime}, f^{\prime \prime}\right)$. This is possible since $\left|\left\{x_{\alpha} \mid \alpha<\beta\right\}\right|<c$.

We now construct an expansion topology for $(I, \varepsilon)$. For every $x \in g(\mathscr{B})$, let $G_{x}=\left[f^{-1}(0) \cap[0, x)\right] \cup\left[f^{-1}(1) \cap(x, 1]\right]$, where $x=g(B)$ and $B=$ $(J, f)$. Let $\mathcal{G}_{x}$ be an ultrafilter of sets $\varepsilon$-singular at $x$ such that $G_{x} \in \mathcal{G}_{x}$. If $y \in I-g(\Re)$, let $\mathcal{S}_{y}$ be an arbitrary ultrafilter of sets $\varepsilon$-singular at $y$. Now set $\mathcal{G}=\cup_{x} \mathcal{G}_{x}$, and let $\sigma=\varepsilon(\mathcal{G})$. Clearly, $\sigma$ is a maximal singular expansion of $\varepsilon$.

We can now show $\sigma$ is connected. By Theorem 2, if there exist $A, B \in \sigma$ such that $A \neq \varnothing \neq B, A \cup B=I$ and $A \cap B=\varnothing$, then $I-\left[\operatorname{Int}_{\varepsilon} A \cup\right.$ $\left.\operatorname{Int}_{\varepsilon} B\right]$ is a Cantor set in $I$. Thus, we may associate with $\{A, B\}$ an element $B_{\alpha}=(J, f)$ such that $\operatorname{Int}_{\varepsilon} A=f^{-1}(0)$ and $\operatorname{Int}_{\varepsilon} B=f^{-1}(1)$. But $x_{\alpha}=g\left(B_{\alpha}\right)$ is a point of $I$ such that $x_{\alpha} \notin J=\operatorname{Int}_{\varepsilon} A \cup \operatorname{Int}_{\varepsilon} B$, and $\mathcal{G}_{x}$ was chosen so that every nbd of $x$ intersects both $A$ and $B$. Thus, no disconnection of $(I, \sigma)$ exists.

TheOREM 4. Let $\delta$ be a submaximal expansion of $(I, \varepsilon)$ and let $\mu$ be $a$ maximal singular expansion of $(I, \delta)$. Then if $\mu$ is connected, $\mu$ is maximal connected.

Proof. Suppose $\mu(A)$ is connected for some $A \subseteq I$. We will show that $A \in \mu$, that is, there is no connected proper expansion of $\mu$. If $A \cap \partial_{\mu} A=\varnothing$, we are done. Thus, let $x \in A \cap \partial_{\mu} A$. Then $x \in A \cap \partial_{\delta} A$, and since $\delta$ is submaximal, there exists $W \in \delta$ such that $W \cap \partial_{\delta} A=\{x\}$. Now ( $W \cap$ $\left.\operatorname{Int}_{\delta} A\right) \in \delta$ and $W \cap \operatorname{Int}_{\delta} A \cap(x, b) \neq \varnothing$ for $b>x$. Otherwise, $[0, x]$ is open and closed in $(I, \mu(A))$. Similarly, $W \cap \operatorname{Int}_{\delta} A \cap(a, x)=\varnothing$. Thus, $(W \cap$ $\left.\operatorname{Int}_{\delta} A\right) \cup\{x\}$ is $\delta$-singular at $x$. Now suppose $V$ is an element of the ultrafilter of sets $\delta$-singular at $x$ which forms a $\mu$-nbd base at $x$. Consider

$$
V \cap\left[\left(W \cap \operatorname{Int}_{\delta} A\right) \cup\{x\}\right]=\left[(V \cap W) \cap \operatorname{Int}_{\delta} A\right] \cup\{x\}
$$

is $\delta$-singular at $x$, else, as above, $I$ is $\mu(A)$-disconnected. Thus, $\left(W \cap \operatorname{Int}_{\delta} A\right)$ $\cup\{x\} \in \mu$. This contradicts $x \in \partial_{\mu} A$; hence, $A \cap \partial_{\mu} A=\varnothing$ and $A \in \mu$.

THEOREM 5. There exists a topology $\mu$ for $R$ such that $\varepsilon \subset \mu$ and $\mu$ is maximal connected. 
Proof. Since $I$ contains a homeomorphic copy of $R$, and connected subspaces of maximal connected spaces are maximal connected, it suffices to show there exists a maximal connected topology for $I$ finer than $\varepsilon$.

By Theorem 3 there exists a connected maximal singular topology $\sigma$ for $I$ such that $\varepsilon \subset \sigma$. Now let $\mu$ be a submaximal expansion of $\sigma$. By Theorem 1 the connected topology $\mu$ can be obtained as a submaximal expansion followed by a maximal singular expansion. Thus, by Theorem $4, \mu$ is maximal connected.

COROLlaRy 5A. Every Euclidean space $R^{n}$ admits a maximal connected expansion.

Proof. Let $R^{n}$ be given the weak topology with respect to the collection of all lines through the origin. By Theorem 5 each line admits a maximal connected topology. The resulting topology for $R^{n}$ is maximal connected.

\section{The construction of Wage.}

LEMMA 6. There exists a topology $\sigma$, on $Q$, that refines the usual topology and is maximal among all topologies having the following property:

$$
\text { If } U \text { is open and } q \in U \text { then }(-\infty, q) \cap U \neq \varnothing \neq(q, \infty) \cap U \text {. }
$$

Proof. Assume that for all $\alpha<\kappa, \sigma_{\alpha}$ is a topology on $Q$ which satisfies (*) and $\sigma_{\beta} \subset \sigma_{\alpha}$ whenever $\beta<\alpha<\kappa$. Let $\sigma_{\kappa}$ be the topology generated by $\bigcup_{\alpha<\kappa} \sigma_{\alpha}$. The lemma will follow from Zorn's lemma once it is shown that $\sigma_{\kappa}$ satisfies (*). If $q \in U \in \sigma_{\kappa}$ then there is an $\alpha<\kappa$ and $V \in \sigma_{\alpha}$ with $q \in$ $V \subset U$. Since $\sigma_{\alpha}$ satisfies (*) and $V \subset U$, we have $(-\infty, q) \cap U \neq \varnothing \neq(q, \infty)$ $\cap U$, and hence $\sigma_{\kappa}$ satisfies (*).

Enumerate as $\left\langle U_{\alpha}: \alpha\left\langle 2^{\omega}\right\rangle\right.$ all clopen elements $U$ of $\sigma$ such that $| \operatorname{cl}_{\varepsilon}(U) \cap$ $\operatorname{cl}_{\varepsilon}(Q-U) \mid=2^{\omega}$. It is not difficult to prove that for each irrational $x$ there are $2^{\omega}$ such clopen sets such that $x \in \operatorname{cl}_{\varepsilon}(U) \cap \mathrm{cl}_{\varepsilon}(Q-U)$. Hence, the irrationals can be inductively ordered as $\left\langle x_{\alpha}: \alpha<2^{\omega}\right\rangle$ so that $x_{\alpha} \in \operatorname{cl}_{\varepsilon}\left(U_{\alpha}\right) \cap$ $\operatorname{cl}_{\varepsilon}\left(Q-U_{\alpha}\right)$ for all $\alpha<2^{\omega}$.

For each $\alpha<2^{\omega}$ choose a set of rationals, $L_{\alpha}$, that lies to the left of $x_{\alpha}$, and a set of rationals, $R_{\alpha}$, that lies to the right of $x_{\alpha}$ such that

(a) $L_{\alpha}, R_{\alpha} \in \sigma$,

(b) $x_{\alpha} \in \operatorname{cl}_{\varepsilon}\left(L_{\alpha}\right) \cap \mathrm{cl}_{\varepsilon}\left(R_{\alpha}\right)$, and

(c) either $L_{\alpha} \subset U_{\alpha}$ and $R_{\alpha} \subset Q-U_{\alpha}$, or $L_{\alpha} \subset Q-U_{\alpha}$ and $R_{\alpha} \subset U_{\alpha}$.

It is easy to find such $L_{\alpha}$ and $R_{\alpha}$ since if we cannot let $L_{\alpha}=\left(-\infty, x_{\alpha}\right) \cap U_{\alpha}$ and $R_{\alpha}=\left(x_{\alpha}, \infty\right) \cap Q-U_{\alpha}$, then just set $L_{\alpha}=\left(-\infty, x_{\alpha}\right) \cap Q-U_{\alpha}$ and $R_{\alpha}=\left(x_{\alpha}, \infty\right) \cap U_{\alpha}$.

Now for each $\alpha<2^{\omega}$, let $\mathcal{L}_{\alpha}$ be a maximal subset of $\sigma$ such that $L_{\alpha} \cap\left(x_{\alpha}\right.$ $\left.-1 / n, x_{\alpha}\right) \in \mathcal{L}_{\alpha}$ for each $n \in \omega$ and $A \cap B \neq \varnothing$ for each $A, B \in \mathcal{L}_{\alpha}$. Similarly define $R_{\alpha}$ in terms of $R_{\alpha}$.

The topology $\rho$. We can now define a basis for a maximal connected $T_{2}$ topology on $R$. The basis contains all members of $\sigma$ plus all sets of the form 
$\left\{x_{\alpha}\right\} \cup L \cup R$ where $\alpha<2^{\omega}, L \in \mathfrak{L}_{\alpha}$ and $R \in \Re_{\alpha}$. Call the topology generated by this basis $\rho$. This new topology is Hausdorff since it is an expansion of the usual topology on the line.

THEOREM 7. $\rho$ is connected.

Proof. Let $U$ be a nonempty clopen element of $\rho$. We will show that $\rho$ is connected by proving that $U$ contains the rationals, and hence all of $R$ (since $Q$ is $\rho$-dense in $R$ ). Suppose that $U$ does not contain $Q$. If it happens that $\left|\mathrm{cl}_{\varepsilon}(Q \cap U) \cap \mathrm{cl}_{\varepsilon}(Q-U)\right|=2^{\omega}$ then $Q \cap U=U_{\alpha}$ for some $\alpha<2^{\omega}$. But by definition the $\rho$-neighborhoods of $x_{\alpha}$ intersect both $U_{\alpha}$ and $Q-U_{\alpha}$ so that $U$ cannot be $\rho$-clopen. Hence, we can assume that $\mid \mathrm{cl}_{\varepsilon}(Q \cap U) \cap \mathrm{cl}_{\varepsilon}(Q-$ $U) \mid<2^{\omega}$. The set $\operatorname{cl}_{e}(Q \cap U) \cap \operatorname{cl}_{\varepsilon}(Q-U)$ is a closed element of the usual topology on $R$ that has cardinality less than $2^{\omega}$ and hence has an $\varepsilon$-isolated point, $x$. (Note that $\operatorname{cl}_{\varepsilon}(Q \cap U) \cap \mathrm{cl}_{\varepsilon}(Q-U) \neq \varnothing$ since $Q \not \subset U$ and $U$ is $\rho$-open and nonempty.) Let $(a, b)$ be an interval in $R$ that witnesses the isolation of $x$. Then (since the interval $(a, x)$ is $\varepsilon$-connected and contains no points of $\left.\operatorname{cl}_{e}(Q \cap U) \cap \mathrm{cl}_{e}(Q-U)\right),(a, x)$ must be contained in either $U$ or $R-U$. Without loss of generality, assume $(a, x) \subset U$. Similarly, either $(x, b) \subset U$ or $(x, b) \subset R-U$. Since $x \in \operatorname{cl}_{\varepsilon}(Q-U)$ and $(a, x) \subset U$, it must be that $(x, b) \subset Q-U$. However, $x$ is in the $\rho$ closure of both $(a, x)$ and $(x, b)$. Hence $U$ is not clopen and it follows that $\rho$ is connected.

\section{THEOREM 8. $\rho$ is maximal connected.}

Proof. To see that $\rho$ is a maximal connected topology assume that $\tilde{\rho}$ is a topology on $R$ that strictly contains $\rho$. If $\tilde{\rho} \cap \mathcal{P}(Q) \neq \sigma$ then, by the maximality of $\sigma$, there is a $q \in Q$ and $A \in \tilde{\rho}$ such that either $(-\infty, q) \cap A=$ $\varnothing$ or $(q, \infty) \cap A=\varnothing$. This implies that either $(-\infty, q)$ or $(-\infty, q]$ is a clopen element of $\tilde{\rho}$ and that $\tilde{\rho}$ is not connected. Thus we can assume that $\tilde{\rho} \cap \mathcal{P}(Q)=\sigma$. Since $\tilde{\rho}$ is strictly larger than $\rho$, there is an $A \in \tilde{\rho}$ and irrational $x_{\alpha}$ such that $x_{\alpha} \in A$ but $A$ contains no $\rho$-neighborhood of $x_{\alpha}$. By intersecting $A$ with a suitable $\rho$-neighborhood of $x_{\alpha}$ we can assume that $A \subset Q \cup\left\{x_{\alpha}\right\}$. Then $A \cap Q \in \sigma$ and, since $A$ is not a $\rho$-neighborhood of $x_{\alpha}$, there is a $U \in \mathfrak{L}_{\alpha} \cup \Re_{\alpha}$ such that $U \cap A=\varnothing$. This implies that either $\left(-\infty, x_{\alpha}\right)$ or $\left(-\infty, x_{\alpha}\right]$ is a clopen element of $\tilde{\rho}$. Thus $\tilde{\rho}$ is not connected and $\rho$ is a maximal connected topology.

5. Concluding remarks. Although the two constructions (actually existence theorems) given here have striking similarities, they are in a sense complementary. The construction of Wage is the more direct, that of Guthrie and Stone the more general. Note, for example, that the Wage example has countable dispersion character (minimum cardinality of an open set). The dispersion character in the Guthrie-Stone example may also be chosen to be countable or, by choosing the ultrafilter of dense sets finer than the filter of sets which are complements of sets of cardinality less than $c$, the dispersion character may be made to remain $c$. 
The following questions remain open:

Question 1. Does there exist a regular $T_{1}$ maximal connected topology?

Question 2. Does there exist a countable maximal connected Hausdorff topology?

Question 3. How may maximal connected Hausdorff topologies be characterized?

It has come to the attention of the authors that P. Simon of Prague has announced a solution to the problem solved in this paper, but we have not been able to obtain a copy of his construction.

\section{REFERENCES}

1. D. R. Anderson, On connected irresolvable Hausdorff spaces, Proc. Amer. Math. Soc. 16 (1965), 463-466. MR 31 \#2700.

2. I. Baggs, A connected Hausdorff space which is not contained in a maximal connected space, Pacific J. Math. 51 (1974), 11-18.

3. C. J. R. Borges, On extensions of topologies, Canad. J. Math. 19 (1967), 474-487. MR 35 \#3621.

4. N. Bourbaki, Elements of mathematics. General topology, Part 1, Addison-Wesley, Reading, Mass., 1966. MR 34 \# 5044a.

5. L. Friedler, Open, connected functions, Canad. Math. Bull. 16 (1973), 57-60.

6. J. A. Guthrie, D. F. Reynolds and H. E. Stone, Connected expansions of topologies, Bull. Austral. Math. Soc. 9 (1973), 259-265. MR 48 \# 7201.

7. J. A. Guthrie and H. E. Stone, Spaces whose connected expansions preserve connected subsets, Fund. Math. 80 (1973), 91-100. MR 48 \#9653.

8. P. C. Hammer and W. E. Singletary, Connectedness-equivalent spaces on the line, Rend. Circ. Mat. Palermo 17 (1968), 245-355. MR 43 \# 8054.

9. M. Katetov, On topological spaces containing no disjoint dense sets, Mat. Sb. 21 (1947), 3-12. MR 9, 98.

10. M. R. Kirch, $A$ class of spaces in which compact sets are finite, Amer. Math. Montly 76 (1969), 42. MR 38 \#3816.

11. R. E. Larson, Maximal connected topologies: a survey of the problem, Notices Amer. Math. Soc. 23 (1976), A-179.

12. N. Levine, Simple extensions of topologies, Amer. Math. Monthly 71 (1964), 22-25. MR 29 \#580.

13. D. F. Reynolds, Preservation of connectedness under extensions of topologies, Kyungpook Math. J. 13 (1973), 217-219.

14. A. K. Steiner, On the lattice of topologies, General Topology and Its Relation to Modern Analysis and Algebra III, Academia, Prague, 1972, pp. 411-415.

15. J. P. Thomas, Maximal connected topologies, J. Austral. Math. Soc. 8 (1968), 700-705. MR 38 \# 5177.

16. ___ Maximal connected Hausdorff spaces, Pacific J. Math. 57 (1975), 581-583.

Department of Mathematics, University of Texas at El Paso, El Paso, Texas 79968

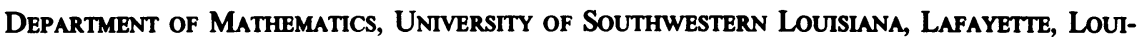
SIANA 70504

InSTITUTE fOR Medicine AND MATHEmatics, OHo University, ATHENS, OHo 45701

Current address (M. L. Wage): Department of Mathematics, Yale University, New Haven, Connecticut 06520 